\section{Prolegómenos}

\section{Derecho y Valores}

Prolegómenos. Derechos y Valores

ISSN: 0121-182X

derechos.valores@umng.edu.co

Universidad Militar Nueva Granada

Colombia

Bueno, Jorge Enrique; Santos Cuevas, Diana Carolina

TEORÍA DE LA AGENCIA EN LA DETERMINACIÓN DE LA ESTRUCTURA DE CAPITAL. CASOS

SECTORES ECONÓMICOS DEL DEPARTAMENTO DEL VALLE DEL CAUCA

Prolegómenos. Derechos y Valores, vol. XV, núm. 30, julio-diciembre, 2012, pp. 161-176

Universidad Militar Nueva Granada

Bogotá, Colombia

Disponible en: http://www.redalyc.org/articulo.oa?id=87625443009

- Cómo citar el artículo

- Número completo

- Más información del artículo

- Página de la revista en redalyc.org

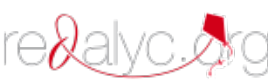

Sistema de Información Científica

Red de Revistas Científicas de América Latina, el Caribe, España y Portugal

Proyecto académico sin fines de lucro, desarrollado bajo la iniciativa de acceso abierto 


\title{
TEORÍA DE LA AGENCIA EN LA DETERMINACIÓN DE LA ESTRUCTURA DE CAPITAL. CASOS SECTORES ECONÓMICOS DEL DEPARTAMENTO DEL VALLE DEL CAUCA*
}

\author{
Jorge Enrique Bueno** \\ Diana Carolina Santos Cuevas***
}

Fecha de recibido: 13 de marzo de 2012

Fecha de aprobación: 12 de junio de 2012

Artículo resultado de investigación

Forma de citación: Bueno, J. E., \& Santos D. C. (2012). La Teoría de Agencia en la determinación de la Estructura de Capital. Caso Sectores Económicos del Departamento del Valle del Cauca. Revista Prolegómenos. Derechos y Valores, 15, 30, 161-176.

\section{Resumen}

El presente artículo se ocupa de la teoría de agencia como determinante de la estructura de capital, considerada esta como un caso especial de la teoría de agencia; lo cual incluye un estudio empírico aplicado a ocho sectores económicos del departamento del Valle del Cauca en el período comprendido entre 2005-2010. La evidencia general sugiere la relevancia de la teoría de agencia en la determinación de la estructura de capital del sector agrícola con predominio exportador.

\section{Palabras clave}

Teoría de agencia, estructura de capital.

\section{THE THEORY OF THE AGENCY IN THE DETERMINATION OF THE CAPITAL STRUCTURE. ECONOMIC SECTORS CASE OF THE VALLE DEL CAUCA DEPARTMENT}

\begin{abstract}
This paper deals with the agency theory as a determinant of capital structure, considered as a special case in agency theory; this includes an empiric study on eight economic sectors pertaining to the department of Valle del Cauca, Colombia, from 2005 to 2010.

Este artículo es el producto del proyecto de investigación "Estructura de capital de algunas empresas corporativas del Valle del Cauca" en la cual el autor principal actúa como coautor, este proyecto es financiado por la Universidad Autónoma de Occidente. Cali. Febrero de 2010 a Enero de 2012.

** Maestría en Economía y Administración, Docente de Planta, Profesional perteneciente al Departamento de Ciencias Económicas de la Universidad Autónoma de Occidente. Cali e investigador del GIED: jebueno@uao.edu.co

**** Economista, Joven investigadora, Universidad Autónoma de Occidente. Grupo GICOF: dianasantosc@hotmail.com
\end{abstract}


The general evidence suggests the relevance of the agency theory indetermining the capital structure of the agricultural sector that focuses on exports.

\title{
Keywords
}

Agency theory, capital structure.

\section{TEORIA DA AGÊNCIA NA DETERMINAÇÃO DA ESTRUTURA DE CAPITAL. SECTORES ECONÔMICOS DO DEPARTAMENTO VALLE DEL CAUCA}

\begin{abstract}
Resumo
Este artigo trata-se da teoria da agência como um determinante da estrutura de capital. Considere a estrutura de capital como um caso especial da teoria da agência. O estudo inclui uma pesquisa empírica aplicado a oito setores econômicos do departamento de Valle del Cauca, no período 2005-2010. A evidência geral sugere a relevância da teoria da agência na determinação da estrutura de capital do setor agrícola de predomínio exportador.
\end{abstract}

\section{Palavras-chaves}

Teoria da agência, estrutura de capital.

\section{INTRODUCCIÓN}

La literatura moderna sobre la estructura de capital tiene su inicio en Modigliani y Miller (1958), en un contexto de competencia perfecta y por lo tanto bajo supuestos, hoy considerados muy restrictivos. La incorporación de imperfecciones tales como los impuestos y costos de quiebras dio paso a un análisis más realista de la estructura de capital. El reconocimiento de las fallas en el modelo de equilibrio general, como consecuencia entre otros aspectos, de la asimetría de información, permitió el desarrollo de la teoría de contratos, que se ocupa del análisis de la interacción de un grupo pequeño de agentes, con información privilegiada, así como de pocos productos.

Desde entonces la literatura económica ha identificado un número amplio de determinantes de la estructura de capital, sin embargo los principios generales que gobiernan los modelos teóricos, son pocos. Uno de ellos, es la relación de agencia.
La relación de agenciaha tomado una renovada notoriedad a raíz de la crisis económica mundial iniciada a mediados de 2007. "Una asunción excesiva de riesgos por parte de los bancos, una serie de conflictos de intereses y un comportamiento fraudulento generalizado, son fenómenos alarmantes que han aparecido repetidamente cuando los mismos han empezado a convertirse en bancarrotas, y la actual crisis no es ninguna excepción" (Stiglitz, 2010, p. 191).

Los estándares regulatorios globales conocidos como Basilea III (Asobancaria, 2011) incluyen entre el conjunto de medidas, mayor transparencia en la información e implementación generalizada de gobierno corporativo por parte de las entidades bancarias, temas cercanos a la relación de agencia.

El reporte de la situación de crédito en Colombia (Banco de la República, 2011) indica que al momento de aprobar nuevos préstamos los principales temas a tener en cuenta por parte de los intermediarios financieros que en Colombia realizan operaciones de crédito y cartera son: 
el conocimiento previo del cliente y su buen historial crediticio, así como el bajo riesgo y las garantías, temas que guardan vinculación con la relación de agencia

El objetivo del presente trabajo es presentar los elementos de la relación de agencia y su vínculocon las dos principales teorías sobre la estructura de capital.Un trabajo empírico aplicado a ocho sectores económicos del Departamento del Valle del Cauca, se incluye. Los aportes fundamentales del presente trabajo son: extender el estudio de agencia en la decisión financiera relacionada con la determinación de la estructura de capital y explorar su relación a nivel sectorial en Colombia.

El documento consta de cinco secciones. En la primera se hace una introducción al tema. La segunda parte presenta los elementos de la relación de agencia, la tercera parte está dedicada a presentar la relación fuentes de financiamiento, estructura de capital y relación de agencia. La sección cuarta se dedica a la evidencia empírica en el caso de sectores económicos del Departamento del Valle del Cauca. Finalmente se presentan las conclusiones.

\section{RELACIÓN DE AGENCIA Y SUS ELEMENTOS}

La relación de agencia se define como un contrato bajo el cual una o más personas llamada(s) principal contrata(n) a otra persona llamada agente para que desempeñe una actividad, la cual involucra una delegación de funciones y responsabilidad al agente y un pago convenido de acuerdo a circunstancias de comportamiento del agente y a los estados de la naturaleza. Si las dos partes tienen comportamientos maximizadores de sus utilidades esperadas y bajo el supuesto de que el agente dispone de mejor información que el principal, existen buenas razones para considerar que el agente no siempre actuará buscando el mejor interés para el principal.

En consecuencia los elementos que se presentan en la relación de agencia son: 1) una relación maximizadora de utilidad para las partes, 2) información asimétrica y 3) un conflicto de intereses. El principal puede limitar el conflicto mediante el establecimiento de incentivos para el agente o mediante mecanismos de monitoreo. Jensen y Meckling (1976) definen los costos de agencia como la suma de 1) los costos de monitoreo (supervisión, restricciones, límites), 2) los costos de vinculación (incentivos) y 3) las pérdidas residuales (reducción de riqueza del principal derivada de los conflictos de interés).

La relación de agencia se presenta en muchas situaciones cotidianas, en el caso empresarial, ejemplos de relaciones de agencia son: relación accionistas- gerente; relación oferente de fondosdemandantes de fondos; gerente- subordinado.

El análisis de los mercados con información asimétrica, importante soporte de la relación de agencia, ha sido ampliamente abordado por George Akerloff (1970), Michael Spence (1973) y Joseph Stiglitz (1981), permitiendo el desarrollo de la teoría de contratos. La existencia de información asimétrica, entre vendedores y compradores de productos, ocasiona que el equilibrio no exista, que no sea sostenible o que no sea económicamente eficiente. Esta idea aplicada al mercado de capitales ocasiona, entre otras consecuencias, el racionamiento del crédito y aplicada a las finanzas corporativas, tiene entre otras aplicaciones, la determinación de la estructura de capital.

La asimetría de información se manifiesta en diversas formas, las principales son:

La selección adversa. Se presenta cuando el agente, debido a su tipología o características, dispone de información privada relevante, antes del inicio de una relación y que obviamente no es conocida por el principal, aunque éste sabe que el agente puede ser de determinada tipología.

Efecto señalización. Es el intento de un participante en una relación, por señalar, antes de firmar el contrato, alguna de sus características relevantes en la relación, y que lo beneficiarían.

Riesgo moral. Se deriva de la realización, después de firmado el contrato, de acciones ocultas por 
parte de un agente económico, de los cuales obtiene beneficios, pero a costa de la contraparte. En este caso el comportamiento del agente no es observable o no verificable por el principal.

La administración de una empresa puede actuar en detrimento de los propietarios de ella, de muchas maneras: insuficiente esfuerzo en el desarrollo de las actividades encomendadas, realizando inversiones extravagantes, adoptando posiciones atrincheradas o mediante un activismo social figurativo para incrementar su perfil. Todas estas actuaciones la literatura especializada las ha rotulado como riesgo moral (moral hazard) Ross (1973) desarrolló la teoría de agencia, nombre con el cual se conoce el riesgo moral o problema del principal-agente.

El esfuerzo se refiere no solo al tiempo dedicado a las labores encomendadas, sino a la elusión de actividades asociadas a la investigación, creatividad, innovación y diseño de estrategias.

Las inversiones extravagantes hacen referencia al emprendimiento de proyectos con la pretensión de mostrar a la empresa en un proceso de crecimiento aunque los proyectos indiquen un VPN negativo.

Las posiciones atrincheradas se refieren a la toma de decisiones encaminadas a asegurar la permanencia del gerente, bien sea emprendiendo líneas de negocios que lo hagan aparecer como indispensable en la organización o mediante la difusión de información indicando que todo va bien.

El activismo social comprende el uso de los recursos de la empresa para incrementar el beneficio privado y la figuración social (jets privados, vinculación a clubes sociales, oficinas excesivamente elegantes y amplias).

\section{FUENTES DE FINANCIAMIENTO: ESTRUCTURA DE CAPITAL Y RELACIÓN DE AGENCIA}

Las fuentes básicas de financiamiento a largo plazo de las corporaciones son: la contratación de deuda, la emisión de capital accionario y las utilidades no distribuidas a los accionistas. En relación con el financiamiento empresarial, (Jensen y Meckling, 1976, pp. 323-328) indicaron la existencia de dos tipos de conflictos de agencia uno entre accionistas y gerentes y otro entre tenedores de deuda y accionistas.

\subsection{Contratación de deuda.}

La relación entre prestamistas (lenders) y prestatarios (borrowers), se ajusta a la definición de relación de agencia. El prestatario (agente) recibe recursos del prestamista(principal). Cuando una empresa adquiere deuda, aparecen conflictos de intereses entre los accionistas(equityholders) y los tenedores de la deuda (debtholders). Los primeros están interesados en maximizar su riqueza y los segundos en la devolución del principal y de los intereses. Específicamente los conflictos surgen debido a que la deuda se constituye en un incentivo para realizar inversiones subóptimas.

Según Jensen y Meckling en ausencia de impuestos y de otras imperfecciones, los costos de agencia asociados con la deuda consisten en: 1) beneficios de oportunidad perdidos por el impacto de la deuda sobre las decisiones de inversión de la firma, 2) gastos de monitoreo y vinculación soportados por los tenedores de bonos y 3) costos de bancarrota y de reestructuraciones.

En opinión de Ross, Westerfield y Jordan (2009) el conflicto entre tenedores de deuda y accionistas surge debido a las estrategias egoístas que los accionistas usan y que perjudican a los tenedores de deuda de la empresa. Las principales estrategias egoístas son:

- Expropiación de valores a los prestamistas, mediante la selección de proyectos riesgosos.

- Al amparo del apalancamiento realizar subinversiones o proyectos con valor presente negativo.

- Ordeño de la propiedad, ya sea mediante dividendos excesivos en épocas de dificultades financieras, lo cual deja una menor cantidad de efectivo para los acreedores. 
Los prestamistas para controlar las anteriores prácticas pueden adoptar dos mecanismos, aumentar las tasas de interés o restringir los créditos. Los prestatarios para evitar cualquiera de las dos situaciones anteriores pueden estar dispuestos a aceptar acuerdos con los prestamistas.

\subsection{Emisión de capital accionario}

La relación entre accionistas y administradores también se ajusta a la definición de relación de agencia. El gerente (agente) recibe recursos de los accionistas(principal), así como autonomía y beneficios monetarios y no monetarios, conjunto de gratificaciones adicionales (perquisites). Es decir obtiene un encargo fiduciario, o cesión de derechos de control. El accionista, léase grupo de accionistas, con derechos legales y económicos plenos, entrega recursos y el gerente debe administrarlos eficientemente. El accionista busca maximizar su riqueza, lo cual es equivalente a maximizar el valor de mercado de la acción de la empresa. El gerente pretende maximizar su bienestar, lo cual está vinculado positivamente al tamaño y crecimiento de la empresa y por lo tanto a las situaciones de poder, prestigio, relaciones, figuración y otros incentivos no monetarios, y negativamente a su esfuerzo. Esta situación origina un conflicto de intereses. La solución del conflicto genera costos de agencia.

Los costos de agencia derivados de la relación accionistas- administradores son amplios y variados, pero se pueden sintetizar en los siguientes:

- Monitoreo, el cual se lleva a cabo por una variedad amplia de agentes y organismos, tales como el consejo de administración o junta directiva, auditoría, acreedores financieros tales como bancos y tenedores de bonos y firmas calificadoras de riesgo.

- Activismo de los inversionistas, lo cual generalmente se ejerce a través de los inversionistas institucionales, particularmente los fondos de pensiones y de cesantías y del mecanismo de propiedad concentrada. En este caso pocosaccionistas con un bloque sustantivo de acciones, tienen alta motivación por el control.

- Paquetes de incentivos monetarios y no monetarios, que motiven al CEO (presidente de la empresa) a pensar y actuar como dueño del negocio.

- Tomas hostiles y compras apalancadas. La amenaza de que la empresa sea adquirida por otra u otras, en un porcentaje que le permita al adquirente asumir el control de la empresa, presiona a los gerentes por un alto desempeño, so pena de perder sus beneficios monetarios y no monetarios

- El ambiente competitivo de la empresa permite establecer un referente de comparación del desempeño de la gestión empresarial, por lo tanto la transparencia en los informes contables, operativos, financieros y sociales, se hace necesaria.

- El endeudamiento, dentro de ciertos límites, actúa como mecanismo disciplinario de la gestión gerencial al limitar la discrecionalidad de los gerentes en el uso del flujo de caja generado por la empresa, controlando que un importe de tal flujo se desvíe hacia usos que benefician al gerente en detrimento de la rentabilidad de los accionistas.

- El Gobierno Corporativo. Conjunto de normas de conducta que regulan las relaciones con las partes interesadas en la empresa (stake holders).

Jensen y Meckling (1976, p. 306) analizan el efecto del financiamiento vía patrimonio, sobre los costos de agencia, comparando el comportamiento del gerente, cuando él es dueño del $100 \%$ de los derechos residuales sobre la firma(no hay separación propiedad-gestión), y cuando él vende una porción de los derechos residuales(hay algún grado de separación propiedad-gestión). En el primer caso, las decisiones del gerente le reportan tanto beneficios monetarios como no monetarios. El equilibrio se obtiene cuando la utilidad marginal de un peso gastado en la obtención de beneficios monetarios, es igual a la utilidad marginal de un peso gastado en la obtención de beneficios no monetarios. 
Si el gerente propietario vende una porción de los derechos residuales, los costos de agencia surgen por la aparición del conflicto de interés entre el gerente y los nuevos accionistas. En este caso la utilidad marginal del gerente se reduce en la medida en la cual él pierde poder por reducción de sus derechos residuales y busca aumentar la utilidad marginal de sus beneficios no monetarios. A medida que se emite más capital accionario, el gerente probablemente aumentará su tiempo de esparcimiento, las prerrogativas relacionadas con el trabajo y las inversiones no rentables.

Harris y Raviv (1991, pp. 297-355) opinan que el conflicto de interés entre accionistas y administradores surge por decisiones administrativas relacionadas con inversión y pago de dividendos. A su vez el conflicto entre tenedores de deuda y accionistas se ocasiona porque la deuda proporciona a los accionistas incentivos para realizar inversiones subótimas. El elemento común de las posiciones de Jensen y Meckling y de Harris y Raviv es la distribución del flujo de caja generado por la operación de la empresa.

Tanto Jensen y Meckling (1986, pp. 323-328) como Harris y Raviv (1991, pp. 297-355) postulan que la deuda mitiga los costos de agencia ya que es un mecanismo de gobernanza.

\subsection{La deuda como mecanismo de gobernanza}

El flujo de caja bruto después de impuestos, generado por la operación de una empresa, se distribuye hacia las inversiones requeridas para continuar con las operaciones normales, atender el servicio de deuda y el pago a los dueños de la empresa. La ausencia de deuda elimina el flujo de caja para los acreedores financieros, pero puede estimular la discrecionalidad del gerente en la distribución del flujo de caja, privilegiando su uso hacia actividades que lo favorezcan personalmente.

Pero también puede mirarse el tema de la deuda y los covenants como una forma de transferencia de derechos de control a inversionistas que, permite la reducción de los ingresos no monetarios para el gerente, aumentando los ingresos pignorables y facilitando el financiamiento externo de la empresa.

Por lo tanto en la relación accionistas- gerente, la deuda como mecanismo de gobernanza es un costo de agencia.

\subsection{Estructura De Capital}

La proporción de las fuentes de financiación respecto al valor de la empresa, determina su estructura de capital. Cada una de estas fuentes se diferencia, desde el punto de vista de la corporación, por el tratamiento tributario que reciben, por su influencia sobre el control de la empresa, por el grado de flexibilidad que le permite a la gestión corporativa, por la influencia de la política monetaria y del mecanismo de transmisión de ésta, vía tasa de interés, por la incidencia de la política cambiaria y del mecanismo de transmisión, vía intervención sobre la tasa de cambio, y obviamente por las expectativas en relación al clima de negocios. Stewart C. Myers (1984, pp. 575-592) contrasta dos formas de abordar la estructura de capital, aclarando que ella transmite información a los inversionistas.

Estructura óptima (tradeoff theory). En este caso la firma tiene un ratio objetivode deuda a valor de la empresa y gradualmente se va moviendo a él, ajustando los dividendos hacia una tasa determinada de pago de dividendos. Las empresas que utilizan esta teoría buscan conseguir un cierto nivel de deuda y patrimonio equilibrando los costos y beneficios derivados de estas dos fuentes de financiamiento. Los beneficios de la deuda son: las ventajas tributarias derivadas de la deducibilidad de los intereses y el hecho de que constituye un mecanismo para disciplinar a la administración de la empresa. Los costos están asociados a la probabilidad de bancarrota y a los costos de agencia.

No existe estructura óptima (pecking order theory). Esta aproximación a la estructura de capital surge de la asimetría de información. Los administradores disponen de mayor información 
sobre las características de la firma, las oportunidades de inversión, los riesgos y los flujos futuros de efectivo, que los inversionistas, anuncios relacionados con estos tópicos representan una transferencia de información de los gerentes a los inversionistas, lo cual afecta la percepción sobre el precio de las acciones. Si esta percepción conduce a un aumento en el precio de las acciones, el valor del patrimonio de la empresa puede quedar subvaluado, por lo cual en el caso de financiación de proyectos con nueva emisión accionaria, los nuevos inversionistas se apropian del mayor valor presente neto de tales proyectos, mientras que los accionistas antiguos salen perjudicados. Para evitar lo anterior el proyecto debe ser financiado con recursos que no sean subvalorados por el mercado, tal como los fondos interno o la deuda no riesgosa. Myers (1984) se refiere a este último procedimiento como pecking order theory.

Las empresas que utilizan esta teoría establecen un orden jerárquico o de prioridades para obtener financiamiento. Prefieren el financiamiento interno al externo y la deuda al patrimonio. La empresa solo se endeudará cuando su generación interna de fondos no alcance para cubrir sus necesidades para la inversión. En otras palabras la empresa acude primero a sus utilidades retenidas, luego a la deuda finalmente a la emisión accionaria.

Como ya se comentó, los gerentes de las firmas conocen más sobre ellas que los inversionistas, de forma tal que los anuncios que hagan en materia de dividendos, inversiones, reestructuraciones, conllevan información que puede modificar el precio de las acciones. Esta asimetría de información afecta la elección entre emisión de deuda y emisión de acciones, y puede privilegiar la teoría del pecking order.

Pero como la decisión de optar por la estructura optima, implica un balance entre los costos y los beneficios de deuda, y como uno de tales costos es los llamados costos de agencia, se deduce que la asimetría de información también puede privilegiar la teoría de del tradeoff.
Frank y Goyal (2004, pp. 219-244) afirman que hay tres estructuras de capital que son particularmente destacables, la teoría del intercambio entre beneficios y costos de la deuda, la teoría de jerarquización o de pecking order y la teoría de sincronización delmercado, según la cual las firmas determinan su estructura de capital de acuerdo a las condiciones del mercado, usan deuda cuando es barato obtenerla y patrimonio cuando el costo de financiación por este medio es bajo.Frank y Goyal examinaron la importancia relativa de varios factores en las decisiones de apalancamiento de firmas públicas en los Estados Unidos, en el período 1950 a 2000. Identificaron los siguientes factores:

- Las firmas pertenecientes a industrias en las cuales la media de endeudamiento es alta, tienden a tener alto apalancamiento.

- Las firmas que tienen una alta razón valor de mercado a valor en libros tienden a tener menos apalancamiento.

- Las firmas que tienen mayor colateral tienden a tener mayor apalancamiento.

- Las firmas con mayores beneficios tienden a tener menor apalancamiento.

- Las firmas que pagan altos dividendos tienden a tener menos apalancamiento que aquellas que pagan más dividendos.

- Firmas grandes tienden a tener alto apalancamiento.

- Cuando las expectativas de alta inflación son altas las firmas tienden a tener alto apalancamiento.

Harris y Raviv (1991, p. 295) identificaron cuatro categorías que determinan la estructura de capital a saber:

- El enfoque de agencia que busca la mitigación de los conflictos entre accionistas, tenedores de deuda y gerentes es decir entre los grupos con derechos sobre la fuente de recursos de la empresa

- El enfoque de asimetría de información, que pretende disminuir los conflictos por medio de la calidad de la información que se hace llegar 
al mercado de capitales, cuando la empresa toma decisiones estratégicas relacionadas con el financiamiento de proyectos o el pago de dividendos.

- El enfoque de la organización industrial según el cual la determinación de la estructura de capital responde al proceso de causalidad estructura- conducta - estrategia de la empresa.

- El enfoque de control y regulación, que se enfoca en los derechos de los grupos de inversionistas

Según Harris y Raviv, en el enfoque de agencia el apalancamiento financiero está relacionado positivamente con: 1) el valor de la firma, 2) la probabilidad de default, 3) el grado de regulación, 4) el flujo de caja libre generado por la empresa, 5) el valor de liquidación y 6) el grado en el cual la firma puede ser objeto de una compra apalancada. Por otro lado el apalancamiento está relacionado negativamente con:1) el grado de oportunidades de crecimiento y 2) con la cobertura de intereses.

Rajan y Zingales (1995, pp. 1421-1460) en su célebre estudio sobre la estructura de capital para grandes firmas de los países pertenecientes al G7, encontraron cuatro factores determinantes del nivel de apalancamiento de las corporaciones en los principales países industrializados.

- La razón de activos fijos a activos totales, como los activos fijos sirven como colateral, facilitan el acceso al endeudamiento al reducir los costos de agencia del endeudamiento asociados a posibilidades de bancarrota.

- La razón valor de mercado a valor en libros, como medida de oportunidades de inversión y de crecimiento. Las firmas con altos ratios, tienden a tener bajos ratios de deuda, ya que pueden enfrentar altos costos de agencia vinculados a posible riesgo de quiebra.

- El tamaño de la firma medido por sus ventas lo cual presupone empresas sólidas, con menores posibilidades de quiebra. Firmas grandes tienden a tener altos ratios de deuda.

- La rentabilidad, como medida de los flujos de caja generados internamente. Firmas rentables tienden a mantener bajos ratios de deuda.

De acuerdo con los resultados de las investigaciones de los autores presentados, la relación de agencia y la asimetría de información son determinantes de la estructura de capital.

\section{MODELO EMPÍRICO}

Teniendo en cuenta las consideraciones teóricas asociadas a la teoría de agencia y su vinculación a la estructura de capital, se llevó a cabo un ejercicio empírico mediante la especificación y estimación econométrica de un modelo sobre la estructura de capital sectorial en Colombia. El modelo, en cuanto a su estructuración, se apoya en los trabajos clásicos ya comentados. El ejercicio sigue el enfoque de la teoría de agencia, en la determinación de la estructura de capital.

\subsection{Características De La Información Utilizada.}

Con información financiera tomada de la Superintendencia de Sociedades, se obtuvo una muestra de empresas pertenecientes a ocho sectores económicos del Departamento del Valle del Cauca, en el período 2005-2010. El detalle de los sectores, el número de empresas $y$ el porcentaje que representa cada sector se muestran en las tablas No 1 y No 2 . 
Tabla 1. Muestra por sectores

\begin{tabular}{|l|c|c|c|c|c|c|c|}
\hline \multicolumn{1}{|c|}{ SECTORES } & 2005 & 2006 & 2007 & 2008 & 2009 & 2010 & $\begin{array}{c}\text { TOTAL POR } \\
\text { SECTOR }\end{array}$ \\
\hline MUESTRA TOTAL & 753 & 728 & 668 & 650 & 725 & 695 & 4219 \\
\hline $\begin{array}{l}\text { AGRICOLA CON } \\
\text { PREDOMINIO } \\
\text { EXPORTADOR }\end{array}$ & 179 & 159 & 165 & 163 & 191 & 185 & 1042 \\
\hline $\begin{array}{l}\text { PRODUCTOS } \\
\text { ALIMENTICIOS }\end{array}$ & 106 & 98 & 87 & 88 & 93 & 93 & 565 \\
\hline $\begin{array}{l}\text { FABRICACION DE } \\
\text { PRENDAS DE VESTIR }\end{array}$ & 49 & 50 & 49 & 44 & 39 & 40 & 271 \\
\hline $\begin{array}{l}\text { MANUFACTURA DE } \\
\text { CALZADO Y PRODUCTOS } \\
\text { RELACIONADO }\end{array}$ & 14 & 13 & 9 & 10 & 9 & 11 & 66 \\
\hline $\begin{array}{l}\text { FABRICACION DE PAPEL, } \\
\text { CARTON Y DERIVADOS }\end{array}$ & 12 & 14 & 14 & 13 & 14 & 14 & 81 \\
\hline $\begin{array}{l}\text { PRODUCTOS DE } \\
\text { PLASTICO }\end{array}$ & 41 & 45 & 43 & 41 & 46 & 43 & 259 \\
\hline $\begin{array}{l}\text { COMERCIO AL POR } \\
\text { MAYOR }\end{array}$ & 339 & 336 & 287 & 280 & 317 & 296 & 1855 \\
\hline $\begin{array}{l}\text { ACTIVIDADES DE } \\
\text { INFORMATICA }\end{array}$ & 13 & 13 & 14 & 11 & 16 & 13 & 80 \\
\hline
\end{tabular}

Fuente: Superintendencia de Sociedades de Colombia. Cálculos propios

Tabla 2. Muestra porcentual por sectores

\begin{tabular}{|l|c|c|c|c|c|c|c|}
\hline \multicolumn{1}{|c|}{ SECTORES } & 2005 & 2006 & 2007 & 2008 & 2009 & 2010 & $\begin{array}{c}\text { TOTAL } \% \\
\text { SECTOR }\end{array}$ \\
\hline $\begin{array}{l}\text { AGRICOLA CON } \\
\text { PREDOMINIO } \\
\text { EXPORTADOR }\end{array}$ & $24 \%$ & $22 \%$ & $25 \%$ & $25 \%$ & $26 \%$ & $27 \%$ & $25 \%$ \\
\hline $\begin{array}{l}\text { PRODUCTOS } \\
\text { ALIMENTICIOS }\end{array}$ & $14 \%$ & $13 \%$ & $13 \%$ & $14 \%$ & $13 \%$ & $13 \%$ & $13 \%$ \\
\hline $\begin{array}{l}\text { FABRICACION DE } \\
\text { PRENDAS DE VESTIR }\end{array}$ & $7 \%$ & $7 \%$ & $7 \%$ & $7 \%$ & $5 \%$ & $6 \%$ & $6 \%$ \\
\hline $\begin{array}{l}\text { MANUFACTURA DE } \\
\text { CALZADO Y PRODUCTOS } \\
\text { RELACIONADO }\end{array}$ & $2 \%$ & $2 \%$ & $1 \%$ & $2 \%$ & $1 \%$ & $2 \%$ & $2 \%$ \\
\hline $\begin{array}{l}\text { FABRICACION DE PAPEL, } \\
\text { CARTON Y DERIVADOS }\end{array}$ & $2 \%$ & $2 \%$ & $2 \%$ & $2 \%$ & $2 \%$ & $2 \%$ & $2 \%$ \\
\hline PRODUCTOS DE PLASTICO & $5 \%$ & $6 \%$ & $6 \%$ & $6 \%$ & $6 \%$ & $6 \%$ & $6 \%$ \\
\hline COMERCIO AL POR MAYOR & $45 \%$ & $46 \%$ & $43 \%$ & $43 \%$ & $44 \%$ & $43 \%$ & $44 \%$ \\
\hline $\begin{array}{l}\text { ACTIVIDADES DE } \\
\text { INFORMATICA }\end{array}$ & $2 \%$ & $2 \%$ & $2 \%$ & $2 \%$ & $2 \%$ & $2 \%$ & $2 \%$ \\
\hline & $100 \%$ & $100 \%$ & $100 \%$ & $100 \%$ & $100 \%$ & $100 \%$ & $100 \%$ \\
\hline
\end{tabular}

Fuente: Superintendencia de Sociedades de Colombia. Cálculos propios 
Metodológicamente se empleó el método de mínimos cuadrados ordinarios y las variables empleadas se detallan a continuación.

\subsubsection{Endeudamiento. Variable dependiente}

En cuanto al endeudamiento existen muchas formas demedirlo, dependiendo de consideraciones de corto o largo plazo, de los ciclos operativos y de efectivo de la empresa y de la política de financiamiento de los activos. En el caso de empresas colombianas el financiamiento a corto plazo, especialmente con proveedores, juega un papel importante en el financiamiento total. Por ello en el presente trabajo se considera razonable utilizar una medición amplia del endeudamiento. Se toma como medida del mismo el total del pasivo sobre el total del activo (TA/TP).

Las variables independientes incluidas son:

\section{- Rentabilidad}

Se toma como medida proxy la utilidad operativa sobre el total de activos (UO/TA). La disponibilidad de fondos generados internamente se captura bien por medidas asociadas a la liquidez o a la rentabilidad de la empresa. Usar fondos generados internamente, reduce la asimetría de la información, elemento de la relación de agencia. Se espera que el coeficiente de esta variable sea negativo.

\section{- Estructura de activos}

La medida proxy escogida es el total del activo no corriente sobre el total del activo(ANC/ TA). El activo no corriente, especialmente el activo fijo, es considerado como el principal colateral que tiene una firma para garantizar la obtención de financiación. Las teorías clásicas sobre estructura de capital tienen posiciones divergentes respecto al signo esperado del coeficiente de esta variable. Para los seguidores de la teoría del tradeoff, existe una relación positiva entre el colateral y el endeudamiento. Para los partidarios de la teoría del pecking order más colateral origina menos propensión a asimetrías de información y por lo tanto menos deuda se emite. Como la asimetría de información es uno de los elementos de la teoría de agencia, este trabajo se acoge a esta postura. Se espera que el coeficiente de esta variable sea negativo.

\section{- Tamaño y solidez}

La variable proxy seleccionada para evaluar el grado de madurez, estabilidad y tamaño se obtiene tomando las ventas sobre el total de activos. El tamaño y solidez de acuerdo con la teoría de agencia, disminuye los costos de agencia de la deuda. Se espera que el coeficiente de esta variable sea positivo.

\section{- Oportunidad de crecimiento}

El margen EBITDA, después de impuestos, indica el porcentaje de cada peso de venta que le queda a una empresa para atender las inversiones requeridas en capital de trabajo, en activos fijos y para atender servicio de deuda y pago de dividendos. Las inversiones están asociadas al crecimiento, por lo tanto el margen EBITDA indica las posibilidades de crecimiento. Como variable proxy del margen EBITDA se ha tomado el margen operativo calculado tomando la utilidad operativa sobre el total de ventas. De acuerdo con la teoría de agencia, el apalancamiento está inversamente relacionado con las oportunidades de crecimiento, ya que los administradores tienen oportunidades de invertir en activos riesgosos a expensas de los prestamistas financieros. Se espera que el coeficiente de esta variable sea negativo.

\section{- Riesgo de default}

Los ratings de deuda calculados por las calificadoras de riesgo tienen en cuenta un conjunto de indicadores, uno de ellos es la cobertura de intereses, calculada como la utilidad operativa sobre el total de gastos financieros. Existe una relación inversa entre bajo margen de cobertura y el endeudamiento. Se espera que el coeficiente de esta variable sea negativo. 


\section{ANÁLISIS DE LOS RESULTADOS}

Para verificar la relación entre la estructura de capital y sus determinantes, se estimó la siguiente ecuación de regresión múltiple:

$Y=B_{o}+B_{1} X_{1 t}+B_{2} X_{2 t}+B_{3} X_{3 t}+B_{4} X_{4 t}+B_{5} X_{5 t}+\mathbf{x}_{t}(1)$

Las tablas No 3 a No 10 resumen los resultados de la aplicación del modelo de mínimos cuadrados. Para el sector agrícola con predominio exportador, observando 2005 y 2010, en términos generales, los signos de los coeficientes de regresión para todas las variables consideradas en el modelo, están en línea con las predicciones de la teoría de agencia y los valores de los estadísticos de prueba son estadísticamente significativos, a un nivel de significancia de $5 \%$ y de $1 \%$. Se exceptúa la variable vinculada a la oportunidad de crecimiento. La variable más importante en la explicación de la estructura de capital es la rentabilidad, sigue en importancia la variable tamaño y solidez.

En el caso del sector comercio al por mayor, para 2005 y 2010 los signos esperados de los coeficientes de regresión de las variables están en general de acuerdo con lo planteado por la teoría de agencia, aunque los valores de los estadísticos de prueba no son totalmente significativos. Las variables más importantes en la explicación de la estructura de capital para este sector son la rentabilidad y la estructura de activos.

En el sector productos alimenticios los signos de los coeficientes, con excepción de la variable posibilidades de crecimiento, están en línea con las predicciones de la teoría de agencia, pero no son estadísticamente significativos. La variable más importante en la explicación de la estructura de capital es la rentabilidad, sigue en importancia la variable estructura de activos.

Para los sectores comentados el estadístico Durbin Watson está cercano a dos (2), lo cual sugiere que no existen problemas de autocorrelación. Desde el punto de vista estadístico el $\mathrm{R}$ cuadrado es bajo pero en comparación con resultados de otras investigaciones se puede considera satisfactorio.

Para los otros sectores, los resultados no indican correspondencia entre la estructura de capital la teoría de agencia.

Tabla 3. Sector agrícola con predominio exportador.

\begin{tabular}{|l|c|c|c|c|}
\hline & \multicolumn{2}{|c|}{2005} & \multicolumn{2}{c|}{2010} \\
\hline \multicolumn{1}{|c|}{ Variable independiente } & b-Coeficiente & T-Value & b-Coeficiente & T-Value \\
\hline Rentabilidad & -1.273498 & $-3.883507 * *$ & -1.317793 & $-3.547789 * *$ \\
\hline Estructura de activos & -0.165873 & $-1.867587 *$ & -0.155075 & $-2.045889 *$ \\
\hline Rotación de activos & 0.228883 & $3.727853^{* *}$ & 0.282625 & $4.32209 * *$ \\
\hline Margen operativo & 0.003708 & 0.238536 & 0.021263 & 0.416748 \\
\hline Cobertura de intereses & -0.000104 & -1.311085 & -0.000268 & $-1.691176^{*}$ \\
\hline & & & & \\
\hline Adj $\mathrm{R}^{2}$ & 0.210147 & & 0.195295 & \\
\hline Durbin-Watson stat & 1.856482 & & 1.528874 & \\
\hline
\end{tabular}

* Nivel de significancia al 5\%

** Nivel de significancia al $1 \%$

Fuente: Los investigadores, Cálculos propios 
Tabla 4. Productos alimenticios

\begin{tabular}{|l|c|c|c|c|}
\hline & \multicolumn{2}{|c|}{2005} & \multicolumn{2}{c|}{2010} \\
\hline \multicolumn{1}{|c|}{ Variable independiente } & b-Coeficiente & T-Value & b-Coeficiente & T-Value \\
\hline Rentabilidad & -1.051223 & $-4.631309 * *$ & -0.114037 & -0.33768 \\
\hline Estructura de activos & -0.539931 & $-4.164873^{* *}$ & -0.325461 & -1.38567 \\
\hline Rotación de activos & 0.025798 & 1.12559 & 0.132406 & $2.740185^{*}$ \\
\hline Margen operativo & 0.00493 & 0.190712 & -0.518646 & -1.253594 \\
\hline Cobertura de intereses & -0.003477 & -0.539613 & -0.000621 & -0.030646 \\
\hline & & & & \\
\hline Adj $\mathrm{R}^{2}$ & 0.345167 & & 0.281754 & \\
\hline Durbin-Watson stat & 1.821967 & & 2.108821 & \\
\hline
\end{tabular}

* Nivel de significancia al $5 \%$

** Nivel de significancia al $1 \%$

Fuente: Los investigadores, Cálculos propios

Tabla 5. Fabricación de prendas de vestir

\begin{tabular}{|l|c|c|c|c|}
\hline & \multicolumn{2}{|c|}{2005} & \multicolumn{2}{c|}{2010} \\
\hline \multicolumn{1}{|c|}{ Variable independiente } & b-Coeficiente & T-Value & b-Coeficiente & T-Value \\
\hline Rentabilidad & 0.335721 & 0.612427 & -0.543799 & -0.762146 \\
\hline Estructura de activos & -0.412918 & $-2.028373^{*}$ & -0.650699 & $-2.312176^{*}$ \\
\hline Rotación de activos & 0.038188 & 0.988925 & -0.00618 & -0.155405 \\
\hline Margen operativo & -0.350135 & -1.468038 & -0.439597 & -1.384878 \\
\hline Cobertura de intereses & -0.018066 & -1.172609 & -0.024106 & -1.165614 \\
\hline & & & & \\
\hline Adj $\mathrm{R}^{2}$ & 0.177822 & & 0.299197 & \\
\hline Durbin-Watson stat & 2.125588 & & 2.2851 & \\
\hline
\end{tabular}

* Nivel de significancia al 5\%

** Nivel de significancia al $1 \%$

Fuente: Los investigadores, Cálculos propios

Tabla 6. Manufactura de calzado

\begin{tabular}{|l|c|c|c|c|}
\hline & \multicolumn{2}{|c|}{2005} & \multicolumn{2}{c|}{2010} \\
\hline \multicolumn{1}{|c|}{ Variable independiente } & b-Coeficiente & T-Value & b-Coeficiente & T-Value \\
\hline Rentabilidad & -1.051556 & -0.451319 & $-3.377797 * *$ & -0.931378 \\
\hline Estructura de activos & -0.492314 & -0.894558 & 0.80724 & 1.135943 \\
\hline Rotación de activos & 0.014087 & 0.068151 & 0.049998 & 0.214631 \\
\hline Margen operativo & 3.084146 & 1.007673 & $3.572829 * *$ & 0.961941 \\
\hline Cobertura de intereses & -0.09295 & -1.301468 & -0.031325 & -1.013729 \\
\hline & & & & \\
\hline Adj $\mathrm{R}^{2}$ & 0.432741 & & 0.56475 & \\
\hline Durbin-Watson stat & 1.777664 & & 1.770319 & \\
\hline
\end{tabular}

* Nivel de significancia al 5\%

** Nivel de significancia al $1 \%$

Fuente: Los investigadores, Cálculos propios 
Tabla 7. Fabricación de papel cartón y derivados

\begin{tabular}{|l|c|c|c|c|}
\hline & \multicolumn{2}{|c|}{2005} & \multicolumn{2}{c|}{2010} \\
\hline \multicolumn{1}{|c|}{ Variable independiente } & b-Coeficiente & T-Value & b-Coeficiente & T-Value \\
\hline Rentabilidad & -4.972641 & -1.263147 & 1.623633 & 0.947515 \\
\hline Estructura de activos & -0.341756 & -1.249524 & -0.207305 & -0.738246 \\
\hline Rotación de activos & 0.577014 & 1.827394 & 0.148088 & 0.812915 \\
\hline Margen operativo & 3.957845 & 1.292684 & -1.320568 & -1.091077 \\
\hline Cobertura de intereses & -0.050363 & -1.006543 & -0.027921 & -0.699031 \\
\hline & & & & \\
\hline Adj $\mathrm{R}^{2}$ & 0.770798 & & 0.649205 & \\
\hline Durbin-Watson stat & 2.696809 & & 1.654603 & \\
\hline
\end{tabular}

* Nivel de significancia al 5\%

** Nivel de significancia al $1 \%$

Fuente: Los investigadores, Cálculos propios

Tabla 8. Productos de plástico

\begin{tabular}{|l|c|c|c|c|}
\hline & \multicolumn{2}{|c|}{2005} & \multicolumn{2}{c|}{2010} \\
\hline \multicolumn{1}{|c|}{ Variable independiente } & b-Coeficiente & T-Value & b-Coeficiente & T-Value \\
\hline Rentabilidad & -0.554133 & -1.154706 & -0.679873 & -1.363527 \\
\hline Estructura de activos & 0.384356 & $2.049697^{*}$ & -0.125903 & -0.56837 \\
\hline Rotación de activos & 0.18653 & $2.039637^{*}$ & -0.006067 & -0.065855 \\
\hline Margen operativo & 0.161766 & 1.848729 & -0.001881 & -0.021196 \\
\hline Cobertura de intereses & -0.010631 & -1.741898 & -0.000715 & -0.770829 \\
\hline & & & & \\
\hline Adj $\mathrm{R}^{2}$ & 0.314589 & & & \\
\hline Durbin-Watson stat & 2.104594 & & & \\
\hline
\end{tabular}

* Nivel de significancia al 5\%

*** Nivel de significancia al $1 \%$

Fuente: Los investigadores, Cálculos propios

Tabla 9. Comercio al por mayor

\begin{tabular}{|l|c|c|c|c|}
\hline & \multicolumn{2}{|c|}{2005} & \multicolumn{2}{c|}{2010} \\
\hline \multicolumn{1}{|c|}{ Variable independiente } & b-Coeficiente & T-Value & b-Coeficiente & T-Value \\
\hline Rentabilidad & -0.119911 & $-2.298136^{*}$ & -0.595208 & $-4.622915^{* *}$ \\
\hline Estructura de activos & -0.252495 & $-3.361601^{* *}$ & -0.265613 & -4.50097 \\
\hline Rotación de activos & 0.019436 & $2.778415^{*}$ & 0.009541 & 1.852903 \\
\hline Margen operativo & -0.895986 & $-29.17534^{* *}$ & 0.05364 & $2.751854^{*}$ \\
\hline Cobertura de intereses & 0.00076 & 0.580791 & -0.007962 & $-3.478793^{* *}$ \\
\hline & & & & \\
\hline Adj $\mathrm{R}^{2}$ & 0.819183 & & 0.214155 & \\
\hline Durbin-Watson stat & 1.99501 & & 1.541224 & \\
\hline
\end{tabular}

* Nivel de significancia al 5\%

** Nivel de significancia al $1 \%$

Fuente: Los investigadores, Cálculos propios 
Tabla 10. Actividades informáticas

\begin{tabular}{|l|c|c|c|c|}
\hline & \multicolumn{2}{|c|}{2005} & \multicolumn{2}{c|}{2010} \\
\hline \multicolumn{1}{|c|}{ Variable independiente } & b-Coeficiente & T-Value & b-Coeficiente & T-Value \\
\hline Rentabilidad & -2.201858 & $-2.162613^{*}$ & -0.760745 & -1.451244 \\
\hline Estructura de activos & -0.422972 & $-2.301238^{*}$ & 0.277501 & 1.304025 \\
\hline Rotación de activos & 0.281502 & 3.036637 & -0.040938 & -0.676534 \\
\hline Margen operativo & 1.89851 & $3.297795^{* *}$ & 0.095468 & $2.387066^{*}$ \\
\hline Cobertura de intereses & -0.061066 & $-3.44393^{* *}$ & $-1.59 \mathrm{E}-05$ & -0.283502 \\
\hline & & & & \\
\hline Adj $\mathrm{R}^{2}$ & 0.778456 & & 0.610976 & \\
\hline Durbin-Watson stat & 1.874912 & & 2.320405 & \\
\hline
\end{tabular}

* Nivel de significancia al $5 \%$

** Nivel de significancia al $1 \%$

Fuente: Los investigadores, Cálculos propios

\section{CONCLUSIONES}

Es conveniente precisar que las decisiones relacionadas con la estructura de capital están influenciadas por muchos determinantes asociados a las estructuras de propiedad, operativa y financiera de las empresas y a sus modelos y estrategias de negocios. También se presentan muchas restricciones particularmente vinculadas con la disponibilidad de información y la heterogeneidad en el tratamiento y clasificación de la información contable.

Entre los principios generales que gobiernan los modelos teóricos sobre estructura de capital de las corporaciones, la relación de agencia ocupa un lugar destacado en los intereses de los investigadores sobre el tema.

Las fuentes de financiamiento a largo plazo de las corporaciones son: la contratación de deuda, la emisión de capital accionario y las utilidades no distribuidas a los accionistas.

La relación entre los agentes de financiamiento y la corporación, se ajusta a la definición de relación de agencia, ya que contiene los elementos fundamentales, a saber: función de utilidad, asimetría de información y conflicto de intereses y sus costos derivados.
La presente investigación analizó ocho sectores económicos del departamento del Valle del Cauca en el período 2005-2010, con el fin de determinar la relevancia de la relación de agencia en la determinación de la estructura de capital de los sectores.

A nivel agregado, de los sectores examinados, solo el sector agrícola con predominio exportador muestra evidencia de una estructura de capital determinada por factores de financiamiento que buscan reducir la asimetría de información y la interpretación de señales por el mercado, derivadas de la revelación de los gerentes de información privilegiada. Por lo tanto el comportamiento del sector se enfoca a aliviar los problemas de agencia. Lo anterior sugiere para el sector una inclinación para privilegiar el financiamiento con recursos internos. La anterior deducción parece razonable habida cuenta de que el sector agrícola con predominio exportador en el departamento del Valle del Cauca, está dominado por los negocios de siembra y cultivo de caña de azúcar y de café, con característica de concentración de propiedad de las empresas que lo componen.

En el caso del sector comercio al por mayor, los hallazgos obtenidos muestran una estructura de capital parcialmente determinada por factores encaminados a reducir los problemas de agencia. 
Los resultados obtenidos para estos dos sectores también reflejan evidencia de que se ajustan mejor con la teoría del peckingorder, es decir que privilegian el financiamiento con utilidades retenidas, mecanismo con baja asimetría de información, respecto a los otros dos mecanismos de financiamiento corporativo, lo cual es consistente con investigaciones realizadas que muestran que esta teoría se acomoda mejor para las empresas grandes y maduras.

Finalmente los resultados para los sectores examinados sugieren que el sustento de la relación ente la teoría de agencia y la estructura de capital requiere, en el caso de los sectores examinados, de información contable y financiera más detallada que permitan identificar más precisamente las variables determinantes asociadas a la asimetría de información.

\section{REFERENCIAS}

Akerloff, G. A. (1970). The market for Lemmons. Quality and the Market Mechanism, Quarterly Journal of Economics, 84, 488-500.

Asobancaria (2011). Implicaciones preliminares de Basilea III en Colombia. Semana Económica, 796, p 1-12.

Banco de la República. (2011). Reporte de la situación de crédito en Colombia. Disponible en:http://www.banrep.gov.co/documentos/ informeseconomicos/encuestas/SCC/2011/ credito_marzo.pdf

Berger, A. N., \& Deyoung, R. (1997). Problem Loans and Cost Efficiency in Commercial Banks. Journal of Banking \& Finance, 21,849-870.

Berle, A.,\& Means, G.(1932).The Modern Corporation and Private Property. Nueva York: Mc Millan.

BrealeyR.;MyersS., \&Allen F. (2008). Principles of Corporate Finance. México, D.F.: McGraw Hill.

Brigham E., \& Houston, J. (2005). Fundamentos de Administración Financiera. Mexico, D.F.: Thomson.
Campbell, J., \& Mackinlay A. (1997). The Econometrics of Financial Markets. Princeton: University Press.

Coase R. H. (1983). The Nature of the Firm, Readings in Price Theory, Homewood, IL, Irwin, New Series, V., 331-351

Fama, E. (1970). Efficient Capital Markets, A Review of Theory and Empirical Work. The Journal of Finance, 25, 2, 383-417.

Frank, M. Z., \&. Goya, V. K. (2004). Capital structure decisions: which factors are reliably important? Journal of Financial Economics, 219-244.

Grinblatt, M., \& Titman. S. (2003). Mercados Financieros y Estrategia Empresarial. Madrid: McGraw Hill.

Harris, M., \& Raviv A.(1991). The Theory of Capital Structure.The Journal of Finance, 46, 1, 297-355.

Jensen, M. C., \& Meckling, W. (1986). Agency Costs of Free Cash Flow, Corporate Finance and Takeovers. Journal of Financial Economics, 76, 2, 323-328.

Jensen, M. C., \& Meckling, W. (1976). Theory of the Firm: Managerial Behavior, Agency Costs and Ownership Estructure. Journal of Financial Economics, 3, 4, 305-360.

Kinga, M. (2007). Determinants of Capital Structure Choice. Evidence from Polish Companies. Published online, Septiembre 2 de 2007, International Atlantic Economic Society.

Matthews, J., \& Thompson, J. (2008). The Economics of Banking. Ciudad: Jhon Wiley \& Sons.

Modigliani. F., \& Miller, M. (1958).The Cost of Capital, Corporation Finance and the Theory of Investment. The American Economic Review,11, 3.

Myers, S. C. (1984). The Capital Structure Puzzle.The Journal of Finance, 39, 3, 575-592. Rajan, G., \& Zingales, L. (1995). What Do We know about Capital Structure. Some Evidence from International Data Journal of Finance, 
50,,5,1421-1460. Ross, S. A., Westerfield, R. W., \& Jordan, B. D.(2010). Fundamentos de Finanzas Corporativas. México: Mc Graw Hill.

Ross, S. A., Westerfield, R. W., \&Jordan, B. D. (2009). Finanzas Corporativas. México: McGraw Hill.

Ross, S. A. (1973). The Economic Theory of Agency: The Principal' Problems. American Economic Review, 57, 134-139.

Sarmiento, R. (2005), La Estructura de Financiamiento de las Empresas. Una Evidencia Teórica y Econométrica para Colombia. 19972004. Documentos de Economía Universidad Javeriana, 16.
Stiglitz,J. (2010). Caída Libre. Madrid: Taurus.

Stiglitz J., \&Weiss, A. (1981). Credit Rationing in Markets with Imperfect Information. The American Economic Review. 71,393-410.

Tenjo, F. et al. (1998). Determinantes de la Estructura de Capital de las Empresas Colombianas (1996-2002). Borradores de Economía, 380.

Varian, H. R. (1992). Análisis Microeconómico. Barcelona: Antoni Bosch.

Tirole, J. (2006). The Theory of Corporate Finance. Princeton: University Press.

Welch, I. (2009). Corporate Finance: an introduction. Prentice Hall. 